\title{
Dessorção e calor isostérico em polpa de manga ${ }^{1}$
}

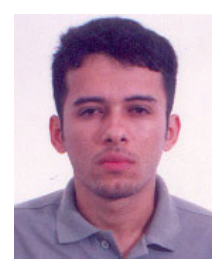

Manassés M. da Silva ${ }^{2}$, Josivanda P. G. de Gouveia ${ }^{3}$ \& Francisco de A. C. Almeida ${ }^{3}$

\author{
1 Parte do trabalho de Iniciação Científica PIBIC/CNPq \\ 2 DEAg/CCT/UFPB. E-mail: manasses.m.s@bol.com.br (Foto) \\ ${ }^{3}$ DEAg/CCT/UFPB. Av. Aprígio Veloso, 882, Bodocongó, CEP 58109-970, Campina Grande, PB. Fone: (83) $310-1287$. \\ E-mail: josi@deag.ufpb.bre diassis@deag.ufpb.br
}

Protocolo $141-20 / 11 / 2000$

\begin{abstract}
Resumo: A polpa da manga (Mangifera indica L.) foi submetida ao processo de dessorção, sob condições de temperatura a 20,40 e $60{ }^{\circ} \mathrm{C}$ e umidade relativa do ar variando de 29,3 a $82,3 \%$, até atingir a umidade de equilíbrio. Posteriormente, cinco modelos matemáticos de sorção (BET, GAB, Halsey, Oswin e Smith) foram ajustados aos dados experimentais com a finalidade de se obter os parâmetros desses modelos e escolher aquele que melhor representasse as isotermas, para o cálculo do calor isostérico de sorção. A escolha do melhor ajuste deu-se em função do coeficiente de determinação $\left(R^{2}\right)$ e do desvio médio relativo $(P)$. De acordo com os resultados o modelo de GAB foi o que melhor se ajustou às isotermas de dessorção e os demais modelos também se ajustaram satisfatoriamente aos dados experimentais, podendo ser empregados para o cálculo da umidade de equilíbrio higroscópico da polpa de manga e, ainda, o calor de sorção variou positivamente de 104,50 a $355,36 \mathrm{~kJ} \mathrm{~kg}^{-1}$.
\end{abstract}

Palavras-chave: isotermas, equilíbrio higroscópico e Mangifera indica L.

\section{Desorption and isosteric heat of mango pulp}

\begin{abstract}
The mango pulp (Mangifera indica L.) has been submitted to the desorption process, at the temperatures of 20,40 and $60{ }^{\circ} \mathrm{C}$, and relative humidity of the air varying from 29.3 to $82.3 \%$ until the equilibrium. Five mathematical models of sorption of hygroscopic products (BET, GAB, Halsey, Oswin and Smith) were adjusted to the experimental data to obtain the coefficients of the models and to choose which one best represented the isotherms for the calculation of the isosteric sorption heat. The choice of the best fit was based on the coefficient of determination $\left(\mathrm{R}^{2}\right)$ and on the relative mean deviation (P). The GAB model was the one which best represented the desorption isotherms, the other models also represented satisfactory the experimental data and may be used for the calculation of the hygroscopic equilibrium humidity of the mango pulp; the sorption heat varied positively from 104.50 to $355.36 \mathrm{~kJ} \mathrm{~kg}^{-1}$.
\end{abstract}

Key words: isotherms, hygroscopic equilibrium and Mangifera indica L.

\section{INTRODUÇÃO}

Para a manga (Mangifera indica L.) o mecanismo de troca de vapor de água entre ela e o ambiente é de grande importância no processamento, particularmente na secagem e armazenagem, por se tratar de um alimento com alto conteúdo de umidade.

Todo alimento contém água, embora esta não se encontre ligada do mesmo modo. Sanchez (1994) e Prado (1998) relatam que, em alguns casos, a água pode estar relativamente livre e, em outros, ligada à estrutura do alimento, não ficando disponível para o processo de deterioração. Assim sendo, o conceito de atividade de água permite que se quantifique o grau de água livre contida no alimento, sendo, portanto, muito importante na preservação de novos produtos alimentícios. Desta forma, a umidade de equilíbrio de um material higroscópico é relevante no estudo da secagem, porque determina o conteúdo de umidade mínimo que o produto pode atingir sob determinadas condições do ar de secagem.

Alguns modelos matemáticos empíricos e teóricos têm sido propostos para o ajuste das curvas de umidade de equilíbrio de vários produtos, em função da atividade de água e também da temperatura do ar, em que, dentre os mais comuns e por sua relativa precisão e generalidade de uso, podem ser citados o de BET, GAB, Halsey, Oswin e Smith (Chirife \& Iglesias, 1978; van der Berg, 1984; Gouveia et al., 1999b). O uso desses modelos é de grande significação tanto no armazenamento quanto na secagem. Durante a secagem e devido às variações contínuas de temperatura e umidade relativa do ar em contato com o produto, ocorrem mudanças no teor de umidade de equilíbrio, sendo, portanto, necessário o seu cálculo inúmeras vezes, os quais são facilitados com o auxílio dessas equações. 
O calor isostérico de sorção é obtido, em geral, a partir de dados de equilíbrio higroscópico e é definido, termodinamicamente, como a diferença entre a entalpia da água na fase de vapor e a entalpia da água líquida adsorvida no sólido, isto é, ele representa a quantidade de energia necessária para evaporar a água adsorvida na fase sólida do produto (Yoshida, 1997). O calor isostérico é um bom parâmetro para se estimar a quantidade mínima de calor requerida para remover uma quantidade de água e permite algumas deduções sobre a microestrutura e as mudanças físicas que acontecem na superfície dos alimentos (Gouveia et al., 1999a). Um método largamente usado para se calcular o calor isostérico de vários alimentos, baseia-se na equação de Clausius-Clapeyron (Iglesias \& Chirife, 1976). Segundo Wang \& Brennan (1991) e Sopade \& Ajisegiri (1994) o conhecimento do calor isostérico em função da umidade de equilíbrio, é essencial nos estudos de secagem e armazenamento de produtos agrícolas, servindo para estimar as necessidades energéticas do processo de secagem fornecendo, também, dados sobre o estado da água no produto. Aqueles autores propuseram uma equação exponencial para descrever a relação entre calor de sorção e o teor de umidade.

Desenvolveu-se este trabalho com o objetivo de: estudar a atividade de água por meio das curvas de dessorção (equilíbrio higroscópico) nas temperaturas de 20,40 e $60^{\circ} \mathrm{C}$, utilizando-se o método gravimétrico estático, numa faixa de umidade relativa de 29,3 a 82,3\%; determinar o calor isostérico a partir dos dados de umidade de equilíbrio e testar cinco modelos matemáticos para definir qual deles apresenta melhor ajuste aos dados experimentais.

\section{MATERIAL E MÉTODOS}

Para determinação do equilíbrio higroscópico em polpa de manga (variedade Tommy Atkins) utilizou-se o método gravimétrico estático com registro descontínuo de mudança de peso. Os experimentos foram realizados no Laboratório de Processamento e Armazenamento de Produtos Agrícolas - LPPA, do Departamento de Engenharia Agrícola da Universidade Federal da Paraíba. O controle das temperaturas $\left(20,40\right.$ e $\left.60^{\circ} \mathrm{C}\right)$ foi garantido por meio de câmaras de circulação forçada de ar, Fanem tipo Demanda Bioquímica de Oxigênio, modelo 347 (Thomas, 1985). As amostras foram colocadas em cadinhos de papel alumínio e levadas em triplicata ao interior de potes herméticos, sendo estes colocados dentro das câmaras com temperaturas fixas de 20,40 e $60^{\circ} \mathrm{C}$. Soluções salinas saturadas foram utilizadas para garantir ambientes com umidade relativa na faixa de 29,3 a $82,3 \%$. Atingido o equilíbrio, as amostras foram levadas à estufa $\left(100^{\circ} \mathrm{C}\right)$ durante $3 \mathrm{~h}$, para determinação da massa seca (AOAC, 1984).

Os ajustes aos dados experimentais dos modelos matemáticos de BET (Eq. 1), GAB (Eq. 2), Halsey (Eq. 3), Oswin (Eq. 4) e Smith (Eq. 5) foram obtidos por meio de regressões não-lineares, utilizando-se o programa de análise estatística STATISTICA versão 5.0. Para o cálculo do calor isostérico $\left(\mathrm{Q}_{\mathrm{ST}}\right)$ fez-se uma regressão linear dos dados do logaritmo neperiano da atividade de água com o recíproco da temperatura a diferentes umidades de equilíbrio e se aplicou a equação de Clausius-Clapeyron (Eq. 6); em seguida, testou-se o modelo exponencial de Sopade e Ajisegiri (Eq. 7) que prediz o comportamento do calor isostérico de sorção em função do conteúdo de umidade de equilíbrio. Equações utilizadas:

BET

$$
\frac{\mathrm{x}}{\mathrm{x}_{\mathrm{m}}}=\frac{\mathrm{C} \mathrm{a}_{\mathrm{w}}}{1-\mathrm{a}_{\mathrm{w}}}\left[\frac{1-(\mathrm{n}+1)\left(\mathrm{a}_{\mathrm{w}}\right)^{\mathrm{n}}+\mathrm{n}\left(\mathrm{a}_{\mathrm{w}}\right)^{\mathrm{n}+1}}{1-(1-\mathrm{C}) \mathrm{a}_{\mathrm{w}}-\mathrm{C}\left(\mathrm{a}_{\mathrm{w}}\right)^{\mathrm{n}+1}}\right]
$$

GAB

$$
\mathrm{x}=\frac{\mathrm{x}_{\mathrm{m}} \mathrm{Ck \textrm {a } _ { \mathrm { w } }}}{\left(1-\mathrm{ka}_{\mathrm{w}}\right)\left(1-\mathrm{k} \mathrm{a}_{\mathrm{w}}+\mathrm{Cka_{ \textrm {w } } )}\right.}
$$

Halsey

$$
a_{w}=\exp \left\{\left[(-a)\left(\operatorname{RT}\left(x / x_{m}\right)^{b}\right)^{-1}\right]\right\}
$$

Oswin

$$
\mathrm{x}=\mathrm{a}\left(\frac{\mathrm{a}_{\mathrm{w}}}{\left(1-\mathrm{a}_{\mathrm{w}}\right)}\right)^{\mathrm{b}}
$$

Smith

$$
\mathrm{x}=\mathrm{M}_{\mathrm{b}}-\mathrm{M}_{\mathrm{a}}\left(\ln \left(1-\mathrm{a}_{\mathrm{w}}\right)\right)
$$

Clausyus-Clayperon

$$
\mathrm{Q}_{\mathrm{ST}}=-\mathrm{R}\left(\frac{\mathrm{d}\left[\ln \left(\mathrm{a}_{\mathrm{W}}\right)\right]}{\mathrm{d}(1 / \mathrm{T})}\right)
$$

Sopade \& Ajisegiri

$$
\mathrm{Q}_{\mathrm{ST}}=\mathrm{A} \exp (\mathrm{Bx})
$$

em que:

$$
\begin{array}{ll}
\mathrm{a}_{\mathrm{w}} & \text { - atividade de água, decimal } \\
\mathrm{n} & \text { - número de camadas moleculares } \\
\mathrm{X} & \text { - conteúdo de umidade de equilíbrio expressa em base } \\
& \text { seca, decimal } \\
\mathrm{X}_{\mathrm{m}} & \begin{array}{l}
\text { - conteúdo de umidade na monocamada molecular, } \\
\text { decimal }
\end{array} \\
\mathrm{C} & \text { - constante de BET relacionada ao calor de sorção da } \\
\mathrm{R} & \text { - camada molecular } \\
\mathrm{T} & \text { - temperatura de realização do experimento, }{ }^{\circ} \mathrm{C} \\
\mathrm{Q}_{\mathrm{ST}} & \text { - calor isostérico, } \mathrm{kJ} \mathrm{kg}^{-1}
\end{array}
$$$$
\text { a, b, } M_{a}, M_{b}, k, A \text { e B - parâmetros de ajuste }
$$

\section{RESULTADOS E DISCUSSÃO}

Os dados da Tabela 1, obtidos experimentalmente, representam a umidade de equilíbrio em função da atividade de água e da temperatura. A esses dados, ajustaram-se os modelos de BET, GAB, Halsey, Oswin e Smith, para se escolher o que melhor representasse o fenômeno de secagem, de modo que os conteúdos de umidade respondessem bem às variações de temperatura. 
Tabela 1. Umidade de equilíbrio (x) para valores de atividades de água $\left(\mathrm{a}_{\mathrm{w}}\right)$ entre 0,290 e 0,820 nas temperaturas de 20,40 e $60^{\circ} \mathrm{C}$

\begin{tabular}{|c|c|c|c|c|c|}
\hline \multicolumn{6}{|c|}{ Temperatura $\left({ }^{\circ} \mathrm{C}\right)$} \\
\hline \multicolumn{2}{|c|}{20} & \multicolumn{2}{|c|}{40} & \multicolumn{2}{|c|}{60} \\
\hline$a_{w}$ & $\mathrm{x}$ & $a_{w}$ & $\mathrm{x}$ & $a_{w}$ & $\mathrm{x}$ \\
\hline 0,331 & 0,062 & 0,316 & 0,034 & 0,293 & 0,022 \\
\hline 0,432 & 0,085 & 0,432 & 0,058 & 0,432 & 0,055 \\
\hline 0,591 & 0,153 & 0,532 & 0,083 & 0,498 & 0,073 \\
\hline 0,699 & 0,253 & 0,661 & 0,120 & 0,631 & 0,107 \\
\hline 0,755 & 0,289 & 0,747 & 0,210 & 0,745 & 0,204 \\
\hline- & - & 0,823 & 0,257 & 0,802 & 0,236 \\
\hline
\end{tabular}

Ponto descartado; Valores de umidade de equilíbrio (x) expressos em base seca

Na Tabela 2 encontram-se os dados que representam os parâmetros dos modelos testados e ajustados para cada uma das temperaturas estudadas, bem como os coeficientes de determinação $\left(\mathrm{R}^{2}\right)$ e os desvios médios relativos $(\mathrm{P})$. De acordo com os resultados, verifica-se que as constantes dos modelos variaram com a temperatura e que o modelo de GAB descreveu com precisão as isotermas de sorção para a atividade de água de 0,293 a 0,823, com valores do coeficiente de determinação variando de 0,988 a 0,995 e desvio médio relativo variando de 0,043 a 0,071, estando de acordo com Lomauro et al. (1985) e Barros Neto et al. (1995) os quais dizem que valores de $\mathrm{R}^{2}$ devem ser próximos da unidade e possuir desvios médios relativos $(\mathrm{P})$ menores que 0,10 .

Tabela 2. Parâmetros de ajuste das isotermas de dessorção em polpa de manga para os diferentes modelos matemáticos

\begin{tabular}{|c|c|c|c|c|c|c|}
\hline \multirow[t]{2}{*}{ Modelo } & \multirow[t]{2}{*}{$\begin{array}{c}\text { Temperatura } \\
\left({ }^{\circ} \mathrm{C}\right)\end{array}$} & \multicolumn{3}{|c|}{ Parâmetros } & \multirow[t]{2}{*}{$\mathrm{R}^{2}$} & \multirow[t]{2}{*}{$\mathrm{P}$} \\
\hline & & $\mathrm{x}_{\mathrm{m}}$ & $\mathrm{C}$ & $\mathrm{n}$ & & \\
\hline \multirow{3}{*}{ BET } & 20 & 0,953 & 0,795 & 4 & 0,994 & 0,056 \\
\hline & 40 & 46,80 & 0,010 & 4 & 0,986 & 0,071 \\
\hline & 60 & 54,19 & 0,008 & 4 & 0,990 & 0,056 \\
\hline \multirow{4}{*}{ GAB } & & $\mathrm{x}_{\mathrm{m}}$ & $\mathrm{C}$ & $\mathrm{k}$ & & \\
\hline & 20 & 5,838 & 0,029 & 0,637 & 0,995 & 0,043 \\
\hline & 40 & 1,624 & 0,058 & 0,687 & 0,988 & 0,048 \\
\hline & 60 & 4,201 & 0,020 & 0,699 & 0,991 & 0,071 \\
\hline \multirow{4}{*}{ Halsey } & & $\mathrm{a}$ & $\mathrm{b}$ & & & \\
\hline & 20 & 0,093 & 0,918 & & 0,982 & 0,042 \\
\hline & 40 & 0,052 & 1,005 & & 0,968 & 0,059 \\
\hline & 60 & 0,062 & 0,909 & & 0,973 & 0,052 \\
\hline \multirow{4}{*}{ Oswin } & & $\mathrm{a}$ & $\mathrm{b}$ & & & \\
\hline & 20 & 0,114 & 0,855 & & 0,992 & 0,035 \\
\hline & 40 & 0,076 & 0,821 & & 0,982 & 0,066 \\
\hline & 60 & 0,071 & 0,891 & & 0,986 & 0,085 \\
\hline \multirow{4}{*}{ Smith } & & $\mathrm{M}_{\mathrm{a}}$ & $\mathrm{M}_{\mathrm{b}}$ & & & \\
\hline & 20 & $-0,018$ & 0,213 & & 0,979 & 0,075 \\
\hline & 40 & $-0,021$ & 0,155 & & 0,966 & 0,095 \\
\hline & 60 & $-0,024$ & 0,154 & & 0,965 & 1,166 \\
\hline
\end{tabular}

De acordo com esses resultados, todos os modelos testados representam satisfatoriamente as isotermas de dessorção em polpa de manga. O modelo de GAB, talvez por ser tri-paramétrico, apresentou o melhor ajuste, resultado este também obtido por Almeida et al. (1999) ao determinarem a umidade de equilíbrio do gergelim (Sesamum indicum L.). Os resultados obtidos a partir dos modelos matemáticos ajustados, estão representados graficamente na Figura 1, que mostra claramente a influência da temperatura sobre a atividade de água do alimento.

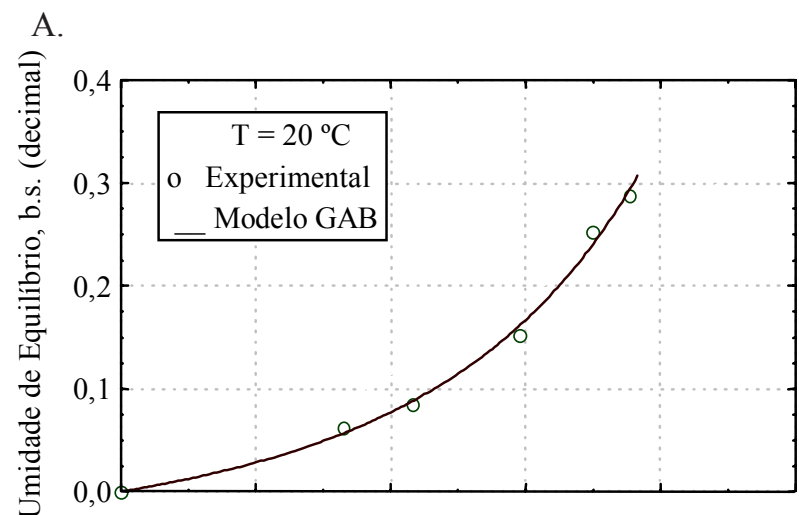

B.

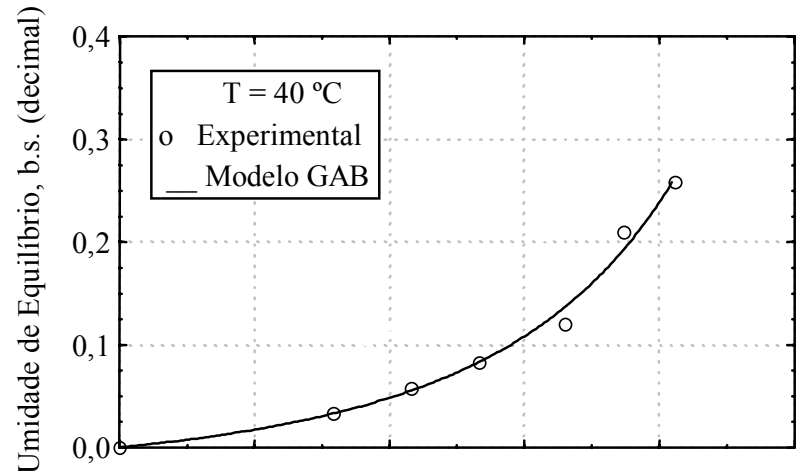

C.

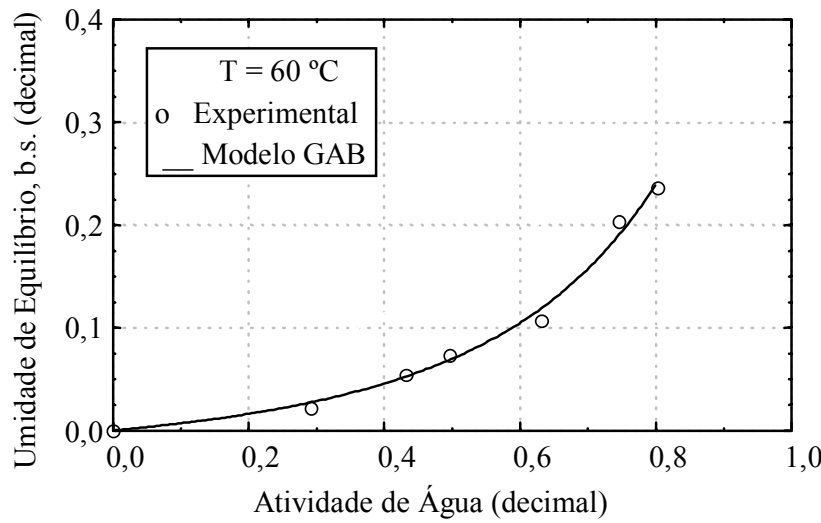

Figura 1. Isotermas de dessorção em polpa de manga nas temperaturas de 20,40 e $60^{\circ} \mathrm{C}$, segundo o modelo GAB

O calor isostérico, ou simplesmente calor de sorção, determinado nesse estudo, é um importante parâmetro a ser obtido porque, por meio dele, é possível se ter uma idéia sobre a demanda energética nos processos de desidratação e secagem de materiais biológicos. As curvas isostéricas de sorção $\left(\ln \left[a_{w}\right]\right)$ versus 1/T (Figura 2) em função da umidade de equilíbrio das amostras, foram obtidas baseadas nos dados das curvas de equilíbrio higroscópico ajustadas pelo modelo de GAB.

O valor do calor isostérico para cada conteúdo de umidade, corresponde ao valor do coeficiente de inclinação da reta, multiplicado pela constante universal dos gases $\left(\mathrm{R}=0,4618 \mathrm{~kJ} \mathrm{~kg}^{-1} \mathrm{~K}^{-1}\right)$ conforme propõe a equação de Clausius-Clapeyron. Esses valores se encontram listados na Tabela 3. 


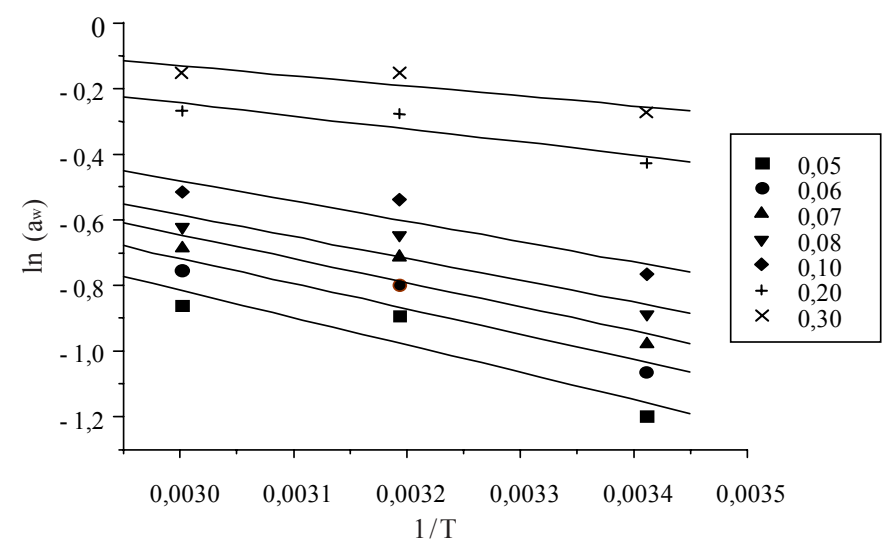

Figura 2. Curvas de $\ln \left(\mathrm{a}_{\mathrm{w}}\right)$ versus $1 / \mathrm{T}$, baseadas na equação de Clausius-Clapeyron

Tabela 3. Valores do calor de sorção da polpa de manga

\begin{tabular}{cc} 
x (b.s.) & Q $_{\text {ST }}\left(\mathrm{kJ} \mathrm{kg}^{-1}\right)$ \\
\hline 0,05 & 355,36 \\
0,06 & 338,82 \\
0,07 & 306,88 \\
0,08 & 286,68 \\
0,10 & 182,94 \\
0,20 & 139,30 \\
0,30 & 104,50 \\
\hline
\end{tabular}

Observando-se os dados da Tabela 3, nota-se decréscimo do calor de sorção com a umidade de equilíbrio, ou seja, quanto menor a umidade da polpa, mais energia é requerida para evaporar a água adsorvida ao produto. Resultado semelhante foi encontrado por Wang \& Brennan (1991) ao secarem batatas em quatro níveis de temperatura. Gouveia et al. (1999a) ao estudarem isotermas de sorção e o calor isostérico do gengibre sem casca, também observaram o mesmo comportamento do calor de sorção, sendo que, neste caso, o calor de sorção do gengibre variou negativamente. Comportamento semelhante também foi observado por Yoshida (1997) ao estudar a cinética de secagem do milho superdoce.

Para o ajuste da curva do calor de sorção $\left(\mathrm{Q}_{\mathrm{ST}}\right)$ em função da umidade de equilíbrio (x), utilizou-se o modelo matemático exponencial de Sopade \& Ajisegiri (1994). Os parâmetros calculados para este modelo, foram $\mathrm{A}=466,51$ e $\mathrm{B}=-6,25$; o coeficiente de determinação $\left(\mathrm{R}^{2}\right)$ e o desvio médio relativo $(\mathrm{P})$

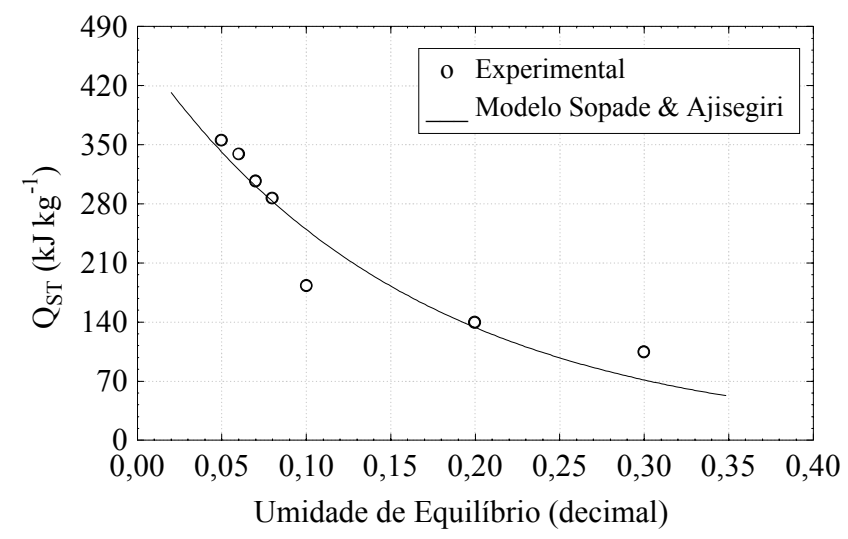

Figura 3. Calor isostérico em função do conteúdo de umidade da polpa de manga, de acordo com a equação de Sopade \& Ajisegiri (1994) para este ajuste foram de 0,900 e 0,120 , respectivamente. Com base nesses valores, pode-se dizer que o modelo prediz, com precisão, os valores do calor de sorção em polpa de manga. O gráfico do calor de sorção em função da umidade encontra-se na Figura 3.

A proporcionalidade inversa do calor de sorção $\left(\mathrm{Q}_{\mathrm{ST}}\right)$ com a umidade de equilíbrio $(\mathrm{x})$ confirma o estudo de Iglesias \& Chirife (1976) que atribuíram este comportamento ao processo de sorção.

\section{CONCLUSÕES}

1. O modelo matemático de GAB foi o que melhor se ajustou às isotermas de dessorção em polpa de manga.

2. Os modelos matemáticos de BET, Halsey, Oswin e Smith, também se ajustaram satisfatoriamente aos dados experimentais, podendo ser utilizados para o cálculo da umidade de equilíbrio higroscópico da polpa de manga.

3. As amostras da polpa de manga entram em equilíbrio higroscópico com valores distintos, dependendo da temperatura a que são submetidas.

4. O calor isostérico de sorção diminui com o aumento da umidade de equilíbrio.

\section{LITERATURA CITADA}

Almeida, F. de A.C.; Fonseca, K.S.; Gouveia, J.P.G. de. Secagem natural de gergelim e determinação da umidade de equilíbrio. Revista Brasileira de Engenharia Agrícola e Ambiental, Campina Grande, PB, v.3, n.3, p.343-348, 1999.

AOAC - Association of Official Analytical Chemists. Official methods of analysis. 14 ed. Arlington, Virginia, 1984, 1v. (s.p.).

Barros Neto, B. de; Scarmínio, I.S.; Bruns, R.E. Planejamento e otimização de experimentos. Campinas, SP: Editora da UNICAMP, 1995.299p.

Chirife, J.; Iglesias, H.A. Equations for fitting water sorption isotherms of foods: Part 1 - A review. Journal of Food Technology, Oxford, v.13, p.159-174, 1978.

Iglesias, H.; Chirife, J. Prediction of the effect of temperature on water sorption isotherms of food material. Journal of Food Technology, Oxford, v.11, p.109-116, 1976.

Gouveia, J.P.G. de; Almeida, F. de A.C.; Fernandez, F.R.; Murr, F.E.X.M. Estudo das isotermas de sorção e calor isostérico do gengibre sem casca. In: Congresso Brasileiro de Engenharia Agrícola, 28, Pelotas, RS. Anais... 1999a. CD Rom

Gouveia, J.P.G. de; Almeida, F. de A.C.; Silva, F.H. da. Estudo da cinética de secagem de gengibre em um secador de leito fixo. Revista Brasileira de Armazenamento, Visçosa, MG, v.24, n.2, p.20-27, 1999b.

Lomauro, C.J.; Bakshi, A.S.; Labuza, T.P. Evaluation of food moisture sorption isotherm equations. Part I: Fruit, vegetable and meat products. Lebensmittel-Wissenschaft und Technologie, Zurich, v.18, n.2, p.111-117, 1985.

Prado, M.E.T. Secagem de tâmaras (Phoenix dactylifera L.) para a obtenção de tâmara passa. Campinas: UNICAMP, 1998. 149p. Tese Doutorado 
Sanchez, C.V. Modelagem e predição da atividade de água em produtos alimentícios. Exame de qualificação de área. Campinas: UNICAMP, 1994. 31p.

Sopade, P.A.; Ajisegiri, E.S. Moisture sorption study on nigerian foods: maize and sorghum. Journal of Food Engineering, Lleida, v.17, n.1, p.33-56, 1994.

Thomas, A.H. Scientific Apparatus and Reagents. Philadelphia: Thomas Scientific. Serving Laboratories the World Over, 1985.2008p. van der Berg, C. Description of water activity of foods for engineering purposes by means of the GAB model of sorption. Engineering and Food, London, v.1, p.311-321, 1984.

Wang, N.; Brennan, J.G. Thermal conductivity of potato as a function of moisture contents. Journal of Food Engineering, Lleida, v.17, n.2, p.153-160, 1991.

Yoshida, C.M.P. Cinética de secagem do milho superdoce. Campinas: UNICAMP, 1997. 149p. Dissertação Mestrado 